\title{
Inventaire et typologie floristique des milieux lentiques dans le district de Vohipeno
}

\author{
Botovao A. Ramiandrisoal, Cyrille Maharombakal, Hery L. \\ T. Ranarijaonal,
}

\begin{abstract}
RÉSUMÉ
L'écologie des macrophytes (plantes aquatiques visibles à l'œil nu) est peu étudiée à Madagascar. L'écosystème aquatique du district de Vohipeno (Région Vatovavy Fitovinany) n'a fait I'objet d'étude que pendant la période coloniale de 1921 à 1936. Les objectifs de cette étude sont d'améliorer la connaissance sur les macrophytes et d'établir une typologie floristique des zones humides malgaches. Au total, 43 espèces réparties entre 34 genres et 19 familles ont été recensées. Les valeurs de l'indice de Shannon Weaver ont montré une faible diversité mais une distribution hétérogène. L'analyse du coefficient de similarité de Sorensen a révélé une ressemblance entre certains sites. L'Analyse Factorielle des Correspondances (AFC) met en exergue quatre groupements de végétaux appuyés par la Classification Ascendante Hiérarchique (CAH).
\end{abstract}

\begin{abstract}
The ecology of macrophytes (aquatic plants) has not been extensively studied in Madagascar. The aquatic ecosystem of the district of Vohipeno (Vatovavy Fitovinany) was last studied during the colonial period 1921 to 1936. The objectives of this study were to improve the knowledge on macrophytes and to make a floristic typology of Malagasy wetlands. In total 43 species distributed between 34 genera and 19 families were recorded. The Shannon Weaver index showed low diversity but a heterogeneous distribution. The Sorensen coefficient analysis revealed resemblances between certain sites. Correspondence Analysis (CA) highlighted four vegetation groups and was supported by Hierarchical Clustering (HCPC).
\end{abstract}

\section{INTRODUCTION}

La flore aquatique malgache est diversifiée tant au niveau familial qu'au niveau spécifique. Elle comprend 338 espèces dont 128 endémiques (Ranarijaona 1999). À titre de comparaison on compte 81 espèces en Australie, 29 espèces en Inde, 116 espèces en Asie, plus de 100 espèces en Europe et plus de 400 espèces dans l'ensemble des zones tropicales (Cook 1996, Ranarijaona 2009). Le recensement le plus récent de Madagascar date de 2017, comprenant 388 espèces, 226 genres et 85 familles (Phillipson et al. 2018). En ce qui concerne l'inventaire floristique des plantes aquatiques (macrophytes) à Vohipeno, le dernier inventaire effectué

\author{
Correspondence: \\ Hery L. T. Ranarijaona \\ Email: hranarijaona@gmail.com
}

date de la période coloniale de 1921 à 1936 selon l'ouvrage « Flore de Madagascar et des Comores » (Humbert 1967). La connaissance sur la flore aquatique de Madagascar est très ancienne. Cependant, à l'exception de cet inventaire, aucune étude n'a été menée sur la flore aquatique du district de vohipeno.

\| existe un lien étroit entre la population locale et les macrophytes. C'est le cas pour l'espèce Typhonodorum lindleyanum, qui sert de nourriture à de nombreuses communautés (Sambo 2010). Les macrophytes constituent une source de revenu notable dans la région de Vohipeno, servant de matières premières pour l'artisanat et la vannerie (fabrication de nattes, chapeaux etc..) et la construction. Mais ce niveau d'exploitation perturbe l'écologie des macrophytes, entrainant la vulnérabilité de leurs écosystèmes (OFEV 2009). La conversion en rizières est le principal facteur de perturbation dans les zones humides à Madagascar (Kull 2012, Bamford et al. 2017, Phillipson et al. 2018) : I'exploitation des zones humides en zone de culture, notamment pour le riz, est très importante et la superficie naturelle des macrophytes régresse. Tous ces faits justifient le choix de la présente étude dont le but est d'améliorer les connaissances sur les macrophytes malgaches à travers des inventaires et des études de typologies des milieux lentiques (eaux stagnantes) du district de Vohipeno. Le district de Vohipeno est caractérisé par une population très dense avec plus de 98,8 hab $/ \mathrm{km}^{2}$ (CREAM 2013) sur une superficie de $1050 \mathrm{~km}^{2}$. Les pressions générées par l'homme influencent les zones humides de Vohipeno. La majorité de la population locale est constituée d'agriculteurs et pousse certaines zones telles que les marais à être converties en rizières. La pêche est une activité relativement importante également et certains lacs sont affectés par la surexploitation. La culture de rente (café, litchi, girofle, etc...) constitue également une source de revenue pour la population locale. Selon la division phytogéographique de Madagascar, la partie orientale de Madagascar (y compris Vohipeno) est caractérisée par une forte pluviométrie pouvant parfois atteindre plus de $3000 \mathrm{~mm}$ (Moat \& Smith 2007). Ces dernières années, de fortes précipitations entraînant des inondations dans cette partie de l'île ont eu des conséquences néfastes pour la production agricole.

\footnotetext{
I Ecole Doctorale Ecosystèmes Naturels, Université de Mahajanga, Mahajanga, Madagascar

॥ Point focal et régional de l'Océan Indien, Sud Expert plantes Développement Durable (SEP2D) et Pôle Recherche et Partenariat Université de Mahajanga

Citation Ramiandrisoa, B.A., Maharombaka, C., Ranarijaona, H.L.T. 2020. Inventaire et typologie floristique des milieux lentiques dans le district de Vohipeno. Madagascar Conservation \& Development 16, S2: p.48-51. http://dx.doi.org/10.4314/mcd.wetlands.7
} 


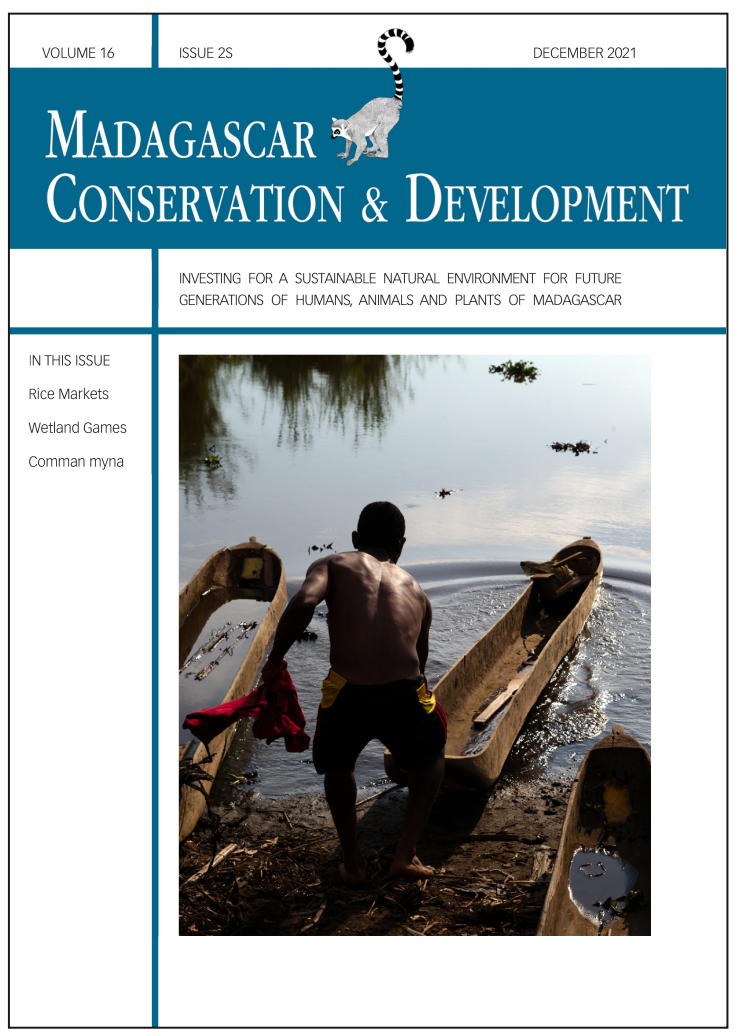

Madagascar Conservation \& Development is the journal of Indian Ocean e-Ink. It is produced under the responsibility of this institution. The views expressed in contributions to MCD are solely those of the authors and not those of the journal editors or the publisher.

All the Issues and articles are freely available at https://www.journalmcd.com

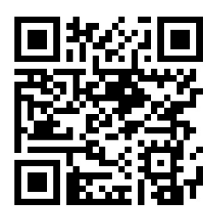

Contact Journal MCD

info@journalmcd.net for general inquiries regarding MCD funding@journalmcd.net to support the journal

Madagascar Conservation \& Development Institute and Museum of Anthropology

University of Zurich

Winterthurerstrasse 190

CH-8057 Zurich

Switzerland

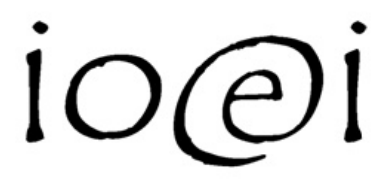

Indian Ocean e-Ink

Promoting African Publishing and Education

www.ioeink.com

Missouri Botanical Garden (MBG)

Madagascar Research and Conservation Program

Missouri Botanical Garden

BP 3391

Antananarivo, 101, Madagascar 


\section{MATÉRIELS ET MÉTHODES}

SITES D'ÉTUDE. Le district de Vohipeno se situe entre E47 $50^{\prime} 25,08^{\prime \prime}, S 22^{\circ} 21^{\prime} 15,264^{\prime \prime}$ dans la province de Fianarantsoa, région Vatovavy Fitovinany (Figure 1). Dix sites ont fait I'objet de cette étude à savoir les marais Ambakamasay (s1), Angaditany (s9), Beafo (s10) et les lacs Andranomavo (s2), Ambato (s3), Ampandroananakara (s4), Alagna (s5), Ekarimbary (s6), Ranomenabe (s7) et Emasomaso (s8). Le climat de la côte sud-est est tropical sous l'influence de l'Alizé. Nos zones d'étude de la commune urbaine de vohipeno et de la commune rurale d'Ivato ont un climat similaire. La période la plus froide est entre le mois de juillet et le mois d'août $\left(16^{\circ} \mathrm{C}\right)$. Le district de Vohipeno est caractérisé par une température moyenne annuelle de $24^{\circ} \mathrm{C}$. La température maximale peut dépasser les $30^{\circ} \mathrm{C}$ entre le mois de décembre et le mois d'avril. Les précipitations annuelles sont abondantes et varient entre 1245 mm et 2504 mm. La pluviométrie est importante du mois de décembre à avril (1230 mm) et généralement faible de juin à novembre (Direction Générale de la Météorologie 2015). Le sol de la région est composé d'alluvion et de sable et représente un terrain de type volcanique du crétacé (FTM 2000). Sur le bas fond, des sols ferralitiques rajeunis à structure plus ou moins dégradée peuvent être distingués, ainsi que des sols anciens indurés et concrétionnées (FTM 2000).

COLLECTES DE DONNÉES. Plusieurs matériels ont été utilisés

pour effectuer les travaux de terrain : un GPS, un appareil photo, de vieux papiers journaux et une presse à herbier. La plante entière a été collectée. Tous les spécimens collectés ont été identifiés dans I'herbarium de Parc Botanique et Zoologique de Tsimbazaza (PBZT), Antananarivo et celui de Mahajanga (Université de Mahajanga). Une vérification des noms scientifiques a été réalisée sur le site web tropicos.org.

RELEVÉ ÉCOLOGIQUE. Chaque relevé a été échantillonné dans un milieu relativement homogène à l'aide d'un quadrat de $1 \mathrm{~m}^{2}$. Dans les relevés échantillonnés, chaque taxon a été désigné par son taux de couverture végétale avec les indices d'abondancedominance adoptés par Braun-Blanquet (1965). Les cinq échelles d'abondance dominance correspondantes sont les suivantes : r) un individu isolé par rapport à la surface échantillonnée ; +) désigne une couverture du taxon faible ; 1) couverture inférieure à $5 \%$;2) couverture entre 5 et $25 \%$; 3) couverture entre 25 à $50 \%$; 4) couverture entre 50 à $75 \%$ et 5) couverture supérieure à $75 \%$.

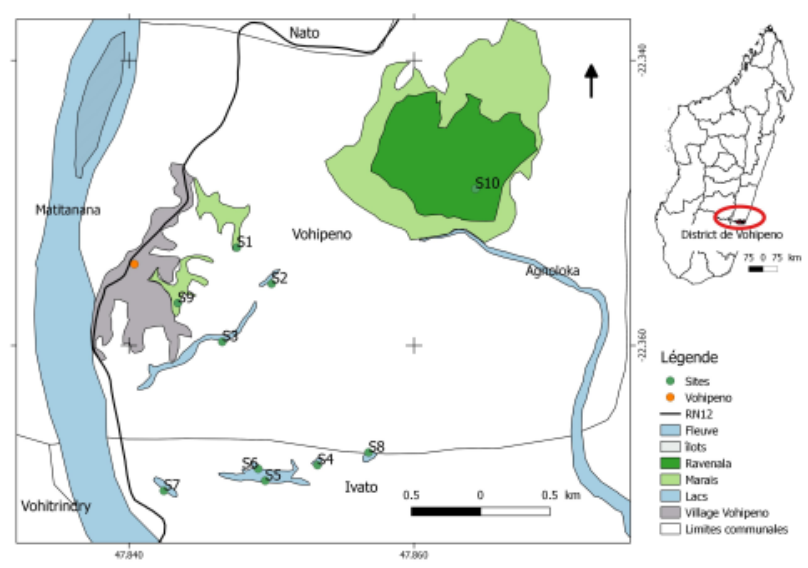

Figure 1. Le district de Vohipeno se situe entre E047 $50^{\prime} 25,08^{\prime \prime}, S 22^{\circ} 21^{\prime} 15,264^{\prime \prime}$ dans la province de Fianarantsoa, région Vatovavy Fitovinany.
INDICE DE DIVERSITÉ. L'indice de Shannon (H') (Shannon 1948,

Shannon \& Weaver 1949), issu de la théorie de l'information, sert à évaluer l'importance de la diversité spécifique dans une station donnée. Cet indice évalue le nombre d’espèces présentes dans un relevé i et tient compte du recouvrement de différentes espèces dans le relevé j. La valeur de cet indice est d'autant plus petite que le nombre de taxon est bas (Vanpeene et al. 1998). La formule suivante permet de calculer ces valeurs.

$$
H^{\prime}=-\sum_{i=1}^{S} p_{i} \log _{2} p_{i}
$$

pi désigne le recouvrement moyen relatif (RM\%) de chaque taxon d'un relevé sur le recouvrement total (RT\%) dans I'ensemble des relevés ( $\mathrm{pi}=\mathrm{RM} \% / \mathrm{RT} \%)$.

L'indice de « régularité » ou "d'équitabilité de Pielou» complète cette approche. Cet indice permet de déterminer si la répartition des espèces est équitable ou au contraire si une seule espèce domine. Cet indice est exprimé par le rapport entre l'indice de diversité de Shannon $\mathrm{H}^{\prime}$ et la diversité maximale $\mathrm{H}^{\prime}$ max. II convient de noter que le logarithme utilisé est le logarithme de base 2.

$$
J^{\prime}=\frac{H^{\prime}}{H^{\prime} \max }
$$

J'étant l'indice de régularité de Pielou

$\mathrm{H}^{\prime}$ max le logarithme de base 2 du nombre de taxon total dans I'ensemble de site.

ÉTUDE COMPARATIVE. Cette étude comparative est la base du test de similarité et permet de déterminer l'affinité floristique entre les sites. Pour permettre le calcul de ce test, la formule suivante a été empruntée à Sorensen (1948).

$$
C s=\frac{2 c}{a+b}
$$

Ce coefficient de similitude « Cs » est le rapport entre le double du nombre d'espèces communes " $\mathrm{C}$ » à deux stations différentes et la somme du nombre total d'espèces des sites « a » et «b ». Si la valeur du coefficient " Cs » dépasse 0,5 les deux sites concernés sont dits similaires.

TRAITEMENTS ET ANALYSES DES DONNÉES. L'objectif de cette analyse est d'identifier les groupements floristiques qui caractérisent un site. Les données brutes ont été arrangées en utilisant Excel puis importées dans le logiciel $\mathrm{R}$ version 3.4.1 pour le traitement. L'AFC a été utilisé pour les données semi-quantitatives (Benzecri 1973, Meddour 2010) avec I'utilisation de code binaire pour chaque taxon (1 si le taxon est présent et 0 sinon). L'AFC (Analyse Factorielle des Correspondances) et la CAH (Classification Ascendante Hiérarchique) sont complémentaires. Le premier explique les différents gradients et le second les scinde et regroupe les sites et les espèces ayant les mêmes caractéristiques écologiques (Benhissoune et al. 2005). Les cartes des sites ont été faites à l'aide du logiciel QGIS version 2.18.

\section{RÉSULTATS}

DIVERSITÉ FLORISTIQUE. Au total, 43 espèces ont été recensées. Elles sont réparties entre 34 genres et 19 familles. Les familles les plus représentées sont les Cyperaceae avec une proportion de $39,5 \%$. Viennent ensuite les Poaceae (11,6\%), 
Araceae (6,9\%), Onagraceae (4,7 \%) et Lentibulariaceae (4,7\%). On trouve 18 familles dans les marais. Elles regroupent 28 genres et 34 espèces. Dix-sept espèces seulement sont typiques des marais (soit 39,5\%). Au niveau des lacs les 26 espèces recensées sont réparties entre 21 genres et 13 familles. Neuf espèces sont typiques des lacs (soit 20,9\%). Le nombre d'espèces communes est de 17 (soit 39,5\%).

INDICE DE DIVERSITÉ. Les valeurs issues de l'indice de Shannon $\left(H^{\prime}\right)$ (Tableau 1) montre que la diversité des plantes aquatiques et semi-aquatiques dans les marais tels qu'Ambakamasay (s1), Beafo (s10) ainsi que dans les lacs tels qu'Alagna (s5) et Ranomenabe (s7) est d'un niveau moyen. En revanche, cette valeur est faible dans d'autres sites, ce qui signifie que leur diversité est faible.

L'indice de régularité de Pielou $\left(J^{\prime}\right)$ est très faible dans le lac Ambato (s3) $\left(\mathrm{J}^{\prime}<0,6\right)$, moyen $\left(0,6<\mathrm{J}^{\prime}<0,8\right)$ dans les lacs et marais des sites s1 (Ambakamasay), s9 (Angaditany), s10 (Beafo), s6 (Ekarimbary) et, enfin, relativement élevé $\left(J^{\prime}>0,8\right)$ dans les sites $s 5$ (Alagna) et s7 (Ranomenabe).

ÉTUDE COMPARATIVE. Le cortège floristique du lac Alagna (s5) montre une affinité dans les sites s6, s7, s8 et s10. Ce dernier a aussi une affinité floristique dans les sites $\mathrm{s1}, \mathrm{s} 5$, s6 et s8. Les espèces communes sont plus nombreuses dans ces sites que dans d'autres.

ANALYSE MULTIVARIÉE. Les deux premiers axes expliquent $45,2 \%$ de l'inertie totale (Figure 2). L'analyse du premier axe (F1 : 25,9\%) permet d'écarter le marais Angaditany (s9, 78,8 \%) sur le côté positif. Le marais est caractérisé par une longue durée d'inondation. Ses espèces caractéristiques sont Azolla pinnata, Marsilea minuta, Commelina diffusa, Lemna paucicostata et Oryza sativa.

L'analyse du second axe factoriel (F2 : 19,4 \%) permet d'isoler deux sites. Le lac Ranomenabe (s7, 23,9\%) situé sur le côté négatif et le marais Ambakamasay (s1, 58,6 \%) sur le côté positif. L'axe F2 détermine le gradient de la profondeur croissante entre les deux sites. Le marais Ambakamasay est caractérisé par des espèces telles que Coix lacryma-jobi, Colocasia esculenta, Blyxa sp, Cyperus pectinatus, Echinochloa pyramidalis, Hydrocotyle vulgaris, Lepironia articulata, Rhynchospora corymbosa et Rhynchospora chinensis.

La classification ascendante hiérarchique (CAH) scinde les sites en quatre groupes. Le premier groupe (groupe A) concerne le marais Angaditany (s9). Ce marais réunit les espèces caractéristiques des zones inondées en permanence. Le second groupe (groupe B) explique la variation de la profondeur du marais Ambakamasay (s1). Le troisième groupe (groupe C) met en évidence les espèces supra aquatiques. Ce sont les espèces qui se rencon-

Table 1. Indice de Shannon-Weaver avec transformation au pourcentage à la médiane de van Der Maarel (1979) du coefficient d'Abondance Dominance de Braun-Blanquet. Les cellules grisées indiquent les valeurs importantes de cette mesure.

\begin{tabular}{lccc} 
Indice & $\mathrm{H}^{\prime}$ & $\mathrm{H}^{\prime} \max$ & $\mathrm{J}^{\prime}$ \\
\hline s1 & 3,2 & 4,46 & 0,72 \\
S2 & 1,26 & 1,58 & 0,79 \\
s3 & 1,1 & 2,58 & 0,43 \\
s4 & 1,55 & 1,58 & 0,98 \\
s5 & 3,29 & 3,58 & 0,97 \\
s6 & 2,45 & 3,58 & 0,68 \\
s7 & 3,62 & 4 & 0,91 \\
s8 & 2,94 & 3,32 & 0,88 \\
s9 & 1,89 & 2,81 & 0,68 \\
s10 & 3,12 & 4,09 & 0,76
\end{tabular}

trent souvent au niveau de la berge des plans d'eaux. Le quatrième groupe (groupe D) réunit les sites où les espèces peuvent coloniser les parties inondées et exondées.

\section{DISCUSSION}

Quarante-trois espèces ont été recensées dans les dix lacs et marais inspectés. Cette richesse est marquée par les espèces appartenant à la famille des Cypreraceae. Cette famille est également plus représentée dans d'autres régions de Madagascar telle que la nouvelle aire protégée d'Antrema, le lac Kinkony (Maharombaka et al. 2012, Ramiandrisoa 2015). Le taxon y est aussi élevé variant de 30 à 50 espèces. Les sites échantillonnés dans le district de Vohipeno possèdent une richesse floristique élevée selon leur indice de diversité biologique. Les valeurs obtenues par l'indice de régularité de Pielou indiquent que les taxons se répartissent plus équitablement d'un site à l'autre, indiquant une hétérogénéité floristique. L'affinité floristique est très significative entre les sites s5, s6, s7, s8 ainsi qu'entre les sites s6 et s7. Cette forte similarité de la composition floristique est due à la faible distance qui sépare ces zones humides. Les zones marécageuses sont plus riches en macrophytes que les zones lacustres. Cependant, le marais Beafo (s10) possède presque tous les taxons rencontrés dans les lacs ainsi que dans le marais Ambakamasay (s1). Ces macrophytes sont distribués selon leurs affinités aux types de substrats ainsi que la situation géographique.

Les lacs ont souvent une surface en eau libre et les macrophytes peuplent leurs berges. Les berges du lac Ranomenabe ont une faible humidité et les espèces qui les caractérisent sont schoenoplectus juncoides et Fimbristylis littoralis. Ces espèces sont souvent rencontrées dans les rizières et sont parfois qualifiées d'adventices. Au niveau des marais, la profondeur de l'eau est variable. Les parties temporairement inondées sont colonisées par des espèces telles que Coix lacryma-jobi, Rhynchospora corymbosa, Lepironia articulata et Hydrocotyle vulgaris. Certaines espèces sont cultivées, comme Colocasia esculenta. D'autres espèces se rencontrent dans les zones un peu plus profondes représentées par des hydrophytes telles que Pistia stratiotes, Lemna paucicostata et Azolla pinnata. Certaines espèces hydrophytes comme Azolla pinnata contribuent à l'enrichissement du substrat en éléments nutritifs notamment en Azote (Roger 1991). La profondeur et la durée d'inondation sont les paramètres déterminant la répartition des espèces du district de Vohipeno. Le marais Angaditany est marqué par une profondeur assez importante. Le lac Ranomenabe met en avant les espèces caractéristiques des berges et des rizières telles que Schoenoplectus juncoides et Fimbristylis littoralis.

Les facteurs influençant la répartition des macrophytes sont relativement similaires dans diverses régions de Madagascar notamment dans la partie orientale de la grande île. La nature du substrat, la profondeur et le taux de composés chimiques de l'eau influencent la distribution des plantes aquatiques (Ranarijaona 2007). La conversion des marais en zone agricole a pris une ampleur considérable comme évoqué par Ranarijaona (2007) et Lammers et al. (2015) sur le lac Alaotra. Ceci est également le cas dans la région Vatovavy Fitovinany particulièrement dans le district de vohipeno.

\section{CONCLUSION}

La richesse floristique est importante dans le district de Vohipeno (région Vatovavy Fitovinany). Au total, 43 espèces ont été recensées. Elles sont réparties entre 34 genres et 19 familles. L'activité 
humaine est aussi importante dans le district étant donné que la majorité de la population locale est composées d'agriculteurs. L'analyse factorielle a permis d'identifier deux facteurs déterminant la répartition des macrophytes des lacs et marais du district de Vohipeno, soit la durée d'inondation et la profondeur. Ces sites d'études cibles n'ont jamais fait l'objet d'inventaire ni d'étude écologique. Cette étude a permis la collecte de nouvelles données ainsi que la mise à jour des connaissances sur la richesse floristique des sites et la répartition géographique des macrophytes.

\section{REMERCIEMENTS}

Nous remercions toute l'équipe qui a contribuée au présent travail, surtout les personnes qui ont participé aux travaux de terrain (guides locaux). Nous remercions également toute l'équipe de I'herbarium TAN du PBZT (Parc Botanique et Zoologique de Tsimbazaza) et celle de I'herbarium de Mahajanga pour leur aide dans l'identification des plantes.

\section{RÉFÉRENCES}

Bamford, A. J., Razafindrajao, F., Young, R. P. \& Hilton, G. M. 2017. Profound and perspective degradation of Madagascar's freshwater wetlands and links with biodiversity. PLOS ONE 12, 8: e0182673. <https://doi.org/10.1371/journal.pone.0182673>

Benhissoune, S., Chaouti, A. et Bayed, A. 2005. Distribution des macrophytes benthiques dans la lagune de Smir (nord-ouest du Maroc). In Écosystèmes Côtiers Sensibles de la Méditerrannée : Cas du Littoral de Smir. Travaux de I'Institut Scientifique, Rabat 4: 27-32. Available online <http://seagrasses.myspecies.info/node/444>

Benzécri, J. P. 1973. L'analyse des Données : L’Analyse des Correspondances. Dunod, Paris.

Braun-Blanquet, J. 1965. Plant Sociology: The Study of Plant Communities. Hafner, Zurich.

Cook, C. D. K. 1996. Aquatic and Wetland plants of India. A Reference Book and Identification Manual for the Vascular Plants found in Permanent or Seasonal Fresh Water in the Subcontinent of India south of the Himalayas. Oxford University Press, Oxford.

CREAM. 2013. Monographie de la région vatovavy Fitovinany. Centre de Recherches d'Études et d'Appui à l'Analyse Économique à Madagascar (CREAM), Région Vatovavy Fitovinany.

FTM. 2000. Foiben-Taosarintanin'i Madagasikara : œuvres. Foiben-Taosarintanin'| Madagasikara (FTM), Antananarive. Available at <https://data.bnf.fr/fr/15158652/foiben-taosarintanin_i_madagasikara/>

Humbert, H. 1967. Flore de Madagascar et des Comores (Plantes Vasculaires). Muséum national d'Histoire naturelle, Laboratoire de Phanérogamie, Paris.

OFEV. 2007. Macrophytes : Instructions pour le Prélèvement d'Échantillons. Méthode d'Analyse et d'Appréciation des Cours d'Eau. Office FDédérale de I'Environnement (OFEV), Ittigen. Available online <shorturl.at/rvH01>

Kull., C. A. 2012. Air photo evidence of historical land cover change in the highlands: Wetlands and grasslands give way to crops and woodlots. Madagascar Conservation \& Development 7, 3: 144-152. $<$ https://doi.org/10.4314/mcd.v7i3.7>

Lammers, P. L., Richter, T., Waeber, P. O. and Mantilla-Contreras, J. 2015. Lake Alaotra wetlands: How long can Madagascar's most important rice and fish production region withstand the anthropogenic pressure? Madagascar Conservation \& Development 10, 3: 116-127. $<$ https://doi.org/10.4314/mcd.v10i3.4>

Maharombaka, C. 2012. Suivi écologique des macrophytes dans le complexe Mahavavy Kinkony. DEA. Université de Mahajanga, Mahajanga.

Meddour, R. 2010. Bioclimatologie, Phytogéographie et Phytosociologie en Algérie. Exemple des Groupements Forestiers et Préforestiers de la Kabylie Djurdjureenne. Thèse. Université Mouloud Mammeri de Tizi Ouzou.

Moat, J. and Smith, P. 2007. Atlas of the Vegetation of Madagascar. Atlas de la Végétation de Madagascar. Kew Publishing, Royal Botanic Gardens, Kew. Available at $<$ shorturl.at/jyHN3>.
Phillipson, P., Andriambololonera, S., Letsara, R. Maharombaka, C., Ramiandrisoa, B. A., et al. 2018. The status and distribution of aquatic plants. In: The status and distribution of freshwater biodiversity in Madagascar and the Indian Ocean Islands Hotspot. L. Máiz-Tomé, C. Sayer, C. \& W. Darwall (eds.), pp 59-74. International Union for the Conservation of Nature (IUCN), Gland, Switzerland. Available online <shorturl.at/fuT09>

Ramiandrisoa, B. A. 2015. Typologie Floristique des Huit Zones Humides de la Nouvelle Aire Protégée d'Antrema. DEA. Université de Mahajanga, Mahajanga.

Ranarijaona, H. L. T., Claude, C. et Gibon, F. M. 2009. Les macrophytes des milieux lentiques de Madagascar : biotypologie, diversité, espèces envahissantes et mesure de conservation. Association Tela Botanica, Montpellier.

Ranarijaona, H. L. T. 1999. Les macrophytes des milieux lentiques de Madagascar (lac, marais et étangs) essais de typologie. Thèse. Université d'Antananarivo, Antananarivo.

Ranarijaona, H. L. T. 2007. Concept de modèle écologique pour la zone humide Alaotra. Madagascar Conservation \& Development 2, $1: 35-42$. <https://doi.org/10.4314/mcd.v2i1.44128>

Roger, P. A. 1993. Les biofertilisants fixateurs d'azote en riziculture : Potentialités, facteurs limitant et perspectives d'utilisation. In: Bas-fonds et Riziculture. M. Raunet (ed.), pp 327-348. CIRAD, Montpellier, France.

Sambo, C. 2010. Les plantes alimentaires sauvages dans la région Anosy (Sud-est de Madagascar). In Les Ignames Malgaches, une Ressource à Préserver et à Valoriser. Acte du Colloque de Toliara. S. Tostain et F. Rejo-Fienena (eds.), pp 108-112. Université de Toliara, Toliara.

Shannon, C. E. 1948. A mathematical theory of communication. The Bell System Technical Journal 27: 379-423, 623-656. <https://doi.org/10.1002/j.1538-7305.1948.tb01338.x>

Shannon, C. E. and Weaver, W. 1949. The Mathematical Theory of Communication. University of Illinois Press, Urbana.

Sorensen, T. 1948. A Method of Establishing Groups of Equal Amplitude in Plant Sociology based on Similarity of Species Content and its Application to Analyses of the Vegetation on Danish Commons. I kommission hos E. Munksgaard, Copenhagen.

Van der Maarel, E. 1979. Transformation of cover-abundance values in phytosociology and its effects on community similarity. Vegetatio 39: 97-114. <http://dx.doi.org/10.1007/BF00052021>

Vanpeene Bruhier, S., Moyne, M. L. et Brun, J. J. 1998. La richesse spécifique : Un outil pour la prise en compte de la biodiversité dans la gestion de l'espace Application en haute Maurienne (Aussois, Savoie). Ingénieries eauagriculture-territoires 15, 12: 47-59. Available online $<$ https://hal.archives-ouvertes.fr/hal-00461198> 